\title{
Determining PPN $\gamma$ with Gaia's astrometric core solution
}

\author{
David Hobbs ${ }^{1}$, Berry Holl ${ }^{1}$, Lennart Lindegren ${ }^{1}$, \\ Frédéric Raison ${ }^{2}$, Sergei Klioner ${ }^{3}$ and Alexey Butkevich ${ }^{3}$ \\ ${ }^{1}$ Lund Observatory, Lund University, Box 43, SE-22100 Lund, Sweden \\ email: david@astro.lu.se \\ ${ }^{2}$ European Space Astronomy Center, ESA, Spain \\ email: frederic.raison@sciops.esa.int \\ ${ }^{3}$ Lohrmann Observatory, Technical University, 01062 Dresden, Germany \\ email: Sergei.Klioner@tu-dresden.de
}

\begin{abstract}
The ESA space astrometry mission Gaia, due for launch in early 2012, will in addition to its huge output of fundamental astrometric and astrophysical data also provide stringent tests of general relativity. In this paper we present an updated analysis of Gaia's capacity to measure the PPN parameter $\gamma$ as part of its core astrometric solution. The analysis is based on small-scale astrometric solutions taking into account the simultaneous determination of stellar astrometric parameters and the satellite attitude. In particular, the statistical correlation between PPN $\gamma$ and the stellar parallaxes is considered. Extrapolating the results to a full-scale solution using some 100 million stars, we find that PPN $\gamma$ could be obtained to about $10^{-6}$, which is significantly better than today's best estimate from the Cassini mission of $2 \times 10^{-5}$.
\end{abstract}

Keywords. Astrometry, relativity, gravitation, data analysis

\section{Introduction}

The space astrometry mission Gaia, planned for launch by the European Space Agency (ESA) in 2012, will determine accurate astrometric data for about 1 billion objects in the magnitude range from 6 to 20. Accuracies of 8-25 micro-arcseconds are expected for the trigonometric parallaxes, positions at mean epoch and annual proper motions of simple (apparently single) stars down to 15th magnitude, increasing to a few hundred $\mu$ as for the faintest objects. To meet the challenge of processing the raw data, the Gaia Data Processing and Analysis Consortium (DPAC) was formed in 2006 (Mignard et al. 2008). A core part of the processing is to determine the accurate spacecraft attitude, geometric instrument calibration and astrometric model parameters for a well-behaved subset of all the objects (the 'primary stars'). In addition, a small number of global parameters will be estimated, one of these being PPN $\gamma$.

Of the 10 parameters used in the PPN formalism (see Will (2006) for a review), Gaia is expected to provide useful constraints on $\beta$ (Hesteroffer et al. 2009), measuring the degree of nonlinearity in the superposition law of gravity, and $\gamma$, measuring the curvature of space time (and hence the degree of gravitational light deflection) due to rest mass. Of interest here is that some alternative theories of gravity predict deviations of these parameters from their values in general relativity $(\gamma=\beta=1)$. In this paper the discussion is restricted to $\gamma$, where deviations of the order $10^{-5}$ to $10^{-8}$ could occur. It is therefore highly interesting to estimate the accuracy by which this parameter could be determined from the Gaia observations. Previous estimates (Mignard 2002; Vecchiato et al. 2003) were based on extrapolations from the Hipparcos results (Frœeschlé et al. 1997) or using 

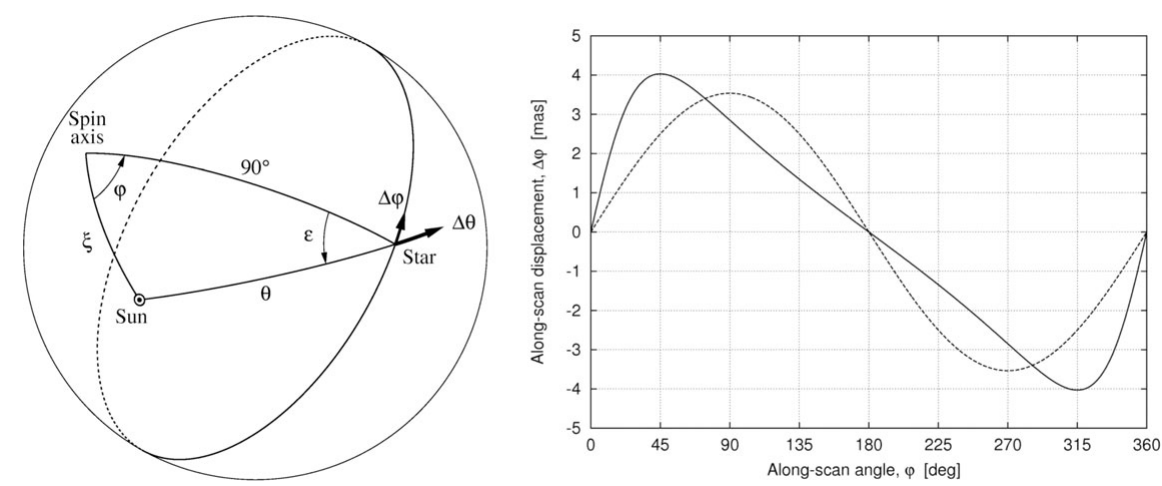

Figure 1. Left: the gravitational deflection by the Sun apparently shifts the star away from the Sun by $\Delta \theta$. Gaia will measure its along-scan component $\Delta \varphi$. Right: the along-scan gravitational deflection (solid curve) together with the effect of a parallax shift by -5 mas (dashed curve).

partly obsolete assumptions about the mission. With the data simulations in DPAC and the core astrometric solution now reaching a high degree of sophistication and realism, more reliable estimates become possible.

\section{The core astrometric solution}

The core astrometric solution is a simultaneous least-squares adjustment of all the different astrometric, attitude, calibration and global parameters to the measured image positions on the CCDs of the primary stars. In total there are more than $5 \times 10^{8}$ unknowns. Although the resulting system of equations is very sparse, a direct solution is unfeasible by many orders of magnitude, and an iterative method must be used. The basic method, known as the Astrometric Global Iterative Solution (AGIS), consists of four blocks executed cyclically until convergence:

- Source Update determines the five astrometric parameters $\left(\alpha, \delta, \varpi, \mu_{\alpha *}, \mu_{\delta}\right)$ for each primary star;

- Attitude Update determines the celestial orientation of the instrument axis as a function of time (using a spline representation of the attitude quaternion);

- Calibration Update determines the geometric instrument calibration parameters (basic angle, CCD geometry, etc.);

- Global Update determines a small number of model parameters that are constant throughout the mission.

In each block, the results of the other three blocks are considered as given. Various schemes can be used to accelerate the convergence of AGIS, but the end result is equivalent to a direct solution of the rigorous normal equations. Since there is no simple way to compute the formal variances of the unknowns, Monte Carlo experiments may be used to 'calibrate' the approximate variances obtained in each block solution.

\section{Measuring gravitational deflection and parallax}

The direct measurement of large angles (of the order of the basic angle, $106.5^{\circ}$ ) is fundamental to Gaia's ability to construct a globally consistent reference system as well as for the determination of parallaxes and $\operatorname{PPN} \gamma$. Gravitational deflection by the Sun causes an apparent shift of a distant object by $\Delta \theta_{\gamma}=(1+\gamma)\left(G M_{\odot} / r c^{2}\right) \cot (\theta / 2)$ in the direction away from the Sun (Fig. 1, left), where $G M_{\odot}$ is the heliocentric gravitational constant, $c$ 
the speed of light, $r \simeq 1.01$ au the heliocentric distance of Gaia, and $\theta$ the Sun-star angle. By contrast, the parallax $\varpi$ causes an apparent shift towards the Sun (actually towards the Solar System Barycenter but this difference is neglected here) by $\Delta \theta_{\varpi}=-\varpi r \sin \theta$. Gaia is mainly sensitive to shifts in the along-scan direction, normal to the nominal spin axis. With $\xi, \varphi$ and $\varepsilon$ defined as in Fig. 1 (left), the along-scan gravitational deflection is $\Delta \varphi_{\gamma}=\Delta \theta_{\gamma} \sin \varepsilon=(1+\gamma)\left(G M_{\odot} / r c^{2}\right) \sin \xi \sin \varphi /(1-\sin \xi \cos \varphi)$, while the along-scan parallax shift is $\Delta \varphi_{\varpi}=\Delta \theta_{\varpi} \sin \varepsilon=-\varpi r \sin \xi \sin \varphi$. The solar aspect angle $\xi=45^{\circ}$ is fixed by the scanning law adopted for Gaia. Resulting variations with $\varphi$ are shown in Fig. 1 (right). To first order, the effect of the gravitational deflection by the Sun is not unlike a global shift of the parallaxes by $\simeq-5$ mas (dashed curve). This correlation between parallax zero point and $\gamma$ was discussed by Mignard (2002) who estimated a correlation coefficient $\rho=0.85$ for the $\xi=55^{\circ}$ then foreseen for Gaia. We find that the correlation coefficient can be analytically evaluated to

$$
\rho=-\left[\int_{0}^{2 \pi} \Delta \varphi_{\gamma}^{2} \mathrm{~d} \varphi \int_{0}^{2 \pi} \Delta \varphi_{\varpi}^{2} \mathrm{~d} \varphi\right]^{-1 / 2} \int_{0}^{2 \pi} \Delta \varphi_{\gamma} \Delta \varphi_{\varpi} \mathrm{d} \varphi=\sqrt{\frac{2 \cos \xi}{1+\cos \xi}}
$$

or $\simeq 0.8538$ for $\xi=55^{\circ}$ in agreement with Mignard. For the current (definitive) Gaia design with $\xi=45^{\circ}$ we find $\rho \simeq 0.9102$. As noted by Vecchiato et al. (2003) this correlation increases the formal standard error for PPN $\gamma$ by a factor $\left(1-\rho^{2}\right)^{-1 / 2}$ compared with a hypothetical solution in which the parallaxes did not have to be determined; unfortunately neglecting parallax would instead result in a huge bias in $\gamma$. With (3.1) we find $\left(1-\rho^{2}\right)^{-1 / 2}=\cot (\xi / 2) \simeq 2.414$ for the current Gaia.

The statistical correlation between PPN $\gamma$ and stellar parallaxes slows down the convergence of the AGIS iterations considerably. To handle this, a global pseudo-parameter $\varpi_{0}$ was introduced in the solution. This $\varpi_{0}$ formally represents a global parallax shift but in the solution it is set to zero immediately after fitting, and therefore does not change the final solution vector in any way. However, it allows the correlation to be taken into account in the Global Update block, resulting in much improved convergence, a better estimate of the formal standard error of $\gamma$, and a numerical estimate of $\rho$ directly from the solution. In each iteration, $\varpi_{0}$ is updated but immediately reset to zero.

\section{Monte Carlo experiments}

Development and testing of the PPN $\gamma$ algorithm uses our simulation software AGISLab (Holl et al. 2009). This tool allows us to perform many independent astrometric solutions in a reasonable time, based on the same principles as the full-scale AGIS (Sect. 2) but using a much smaller number of primary stars, along-scan measurements only, and several other time-saving simplifications (e.g., Calibration Update is not included for the present experiments). The scaling is designed to allow reliable extrapolation to the fullscale solution by preserving key parameters such as the average number of primary stars in the field of view.

For the present experiments the following simple model (Lindegren et al. 1992) was used to calculate the apparent star direction $\hat{\mathbf{u}}$ (ignoring stellar aberration) and its partial derivative with respect to $\gamma$ (angular brackets denoting vector normalization):

$$
\hat{\mathbf{u}}=\left\langle\overline{\mathbf{u}}+\mathbf{r} \frac{(1+\gamma) G M_{\odot} c^{-2}}{r(r+\overline{\mathbf{u}} \cdot \mathbf{r})}\right\rangle \quad \frac{\partial \hat{\mathbf{u}}}{\partial \gamma}=(\mathbf{r}-\overline{\mathbf{u}}(\overline{\mathbf{u}} \cdot \mathbf{r})) \frac{G M_{\odot} c^{-2}}{r(r+\overline{\mathbf{u}} \cdot \mathbf{r})}
$$

Here $\overline{\mathbf{u}}$ is the star's coordinate direction and $\mathbf{r}$ the heliocentric position of Gaia. Gravitational light deflection due to the planets is not included. 


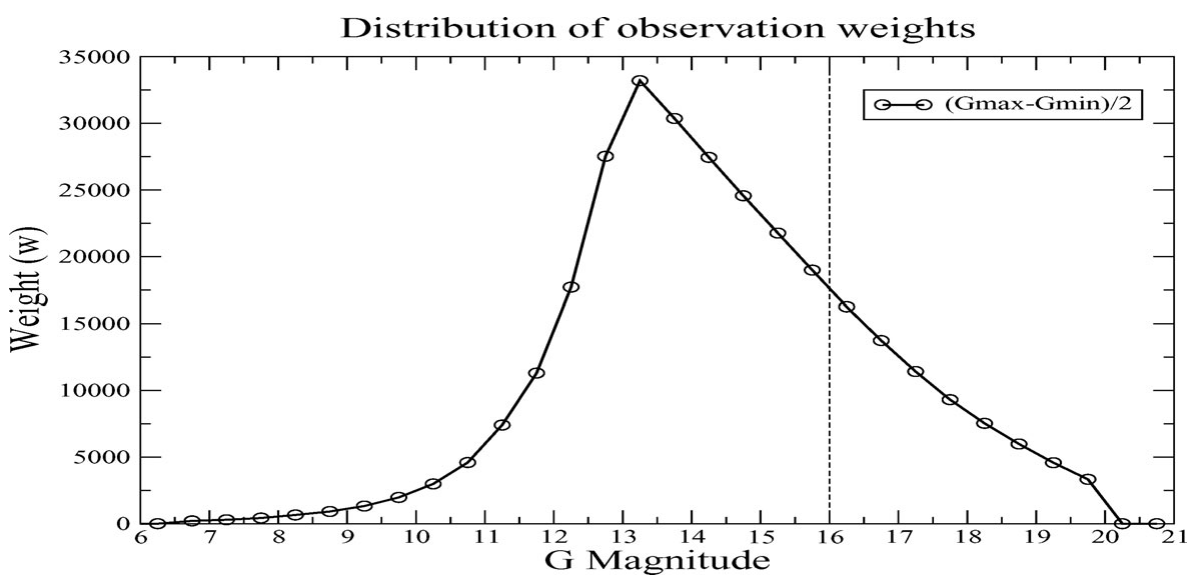

Figure 2. The distribution of observation weights for each $G$-magnitude bin (0.5) clearly shows that primary stars with $G \leqslant 16$ contribute most to the determination of PPN $\gamma$. The figures assume that only $10 \%$ of the measured stars are used.

Using AGISLab, we have run large numbers (typically $M=100$ ) of solutions with different initial conditions, random star distributions and observation noises, and calculated the resulting PPN $\gamma$ (assuming $\gamma=1$ when generating the observations) and its formal standard error $\sigma_{\gamma}^{\mathrm{Lab}}$ in the Global Update (which takes into account the correlation between $\gamma$ and $\varpi_{0}$ ). Based on simulations with $N=10000$ and 50000 primary stars, it was verified that the formal standard error $\sigma_{\gamma}$ as well as the sample standard deviation of the many different estimates of $\gamma$ scale exactly as $w^{-1 / 2}$, where $w=\sum_{\text {obs }} \sigma_{\text {obs }}^{-2}$ is the total statistical weight of the observations used in the solution. Here, $\sigma_{\mathrm{obs}}$ is the assumed along-scan standard error of a single observation, resulting from the crossing of one primary star over one CCD in the astrometric field of Gaia. In the real Gaia, $\sigma_{\mathrm{obs}}$ is mainly a function of the $G$ magnitude of the star (with $G$ representing the very broad spectral response of Gaia, $\simeq 330-1000 \mathrm{~nm}$ ), and has been estimated through extensive Monte Carlo simulations of the image location process using the Gaia Accuracy Analysis Tool (de Bruijne 2005). For bright stars $(G=6$ to 13$)$ this gives $\sigma_{\text {obs }}=75 \mu$ as assuming that the CCDs can be operated near full-well capacity for these stars; the corresponding numbers at $G=15$ and 20 are $240 \mu$ as and 3.1 mas, respectively.

The small-scale simulations using AGISLab are thus used to calculate statistics for the distribution of errors in the estimated PPN $\gamma$ which can then be extrapolated to the full-scale AGIS solution. The extrapolation uses the following formula:

$$
\sigma_{\gamma}^{\text {Full }}=U \times \sigma_{\gamma}^{\mathrm{Lab}} \times\left(\frac{w^{\mathrm{Full}}}{w^{\mathrm{Lab}}}\right)^{-\frac{1}{2}}, \quad U=\sqrt{\frac{1}{M} \sum_{i=1}^{M}\left(\frac{\gamma_{i}-1}{\sigma_{\gamma i}^{\mathrm{Lab}}}\right)^{2}}
$$

Here, the labels Full and Lab refer to the full-scale AGIS and the small-scale AGISLab solutions, respectively, and $\gamma_{i}$ and $\sigma_{\gamma i}^{\mathrm{Lab}}$ are the estimated value in the $i$ th solution and its formal standard error. $U$ is an empirical factor correcting for the neglected correlations between $\gamma$ and all other unknowns except $\varpi_{0}$.

\section{Results and discussion}

A typical Monte Carlo experiment consisted of 100 simulated solutions of 10000 primary stars, each using on average 8768000 observations with an assumed observation 
noise of $\sigma_{\text {obs }}=75 \mu \mathrm{as}$; thus $w^{\text {Lab }}=1559 \mu \mathrm{as}^{-2}$. From several such experiments we derived $U=1.08 \pm 0.08$ with no significant variation between experiments. The mean formal standard error was $\sigma_{\gamma}^{\mathrm{Lab}}=4.61 \times 10^{-5}$. The mean correlation coefficient between $\gamma$ and $\varpi_{0}$ was $\rho=0.9086$, in good agreement with (3.1).

The main uncertainty in (4.2) comes from $w^{\text {Full }}$, which depends strongly both on the assumed number of primary stars used in AGIS and their distribution in magnitude. The final AGIS solution will use at least 100 million primary stars $(10 \%$ of the total number of stars with $G \leqslant 20$ ), but their distribution in magnitude is not fixed. Binaries and other problematic objects must be filtered out, but the fraction of 'well-behaved' stars remaining is likely to be much greater than $10 \%$.

Figure 2 shows the distribution of the weight of all potential observations in halfmagnitude bins of $G$. For $G<13, \sigma_{\text {obs }}$ is roughly constant and the weight per bin increases in proportion to the number of stars. For fainter stars, the increased photon noise is only partially offset by the larger number of stars. Since the magnitude range up to $G \simeq 16$ contains about 100 million stars, the maximum possible number of primary stars should be selected in this interval. For example, assuming that $80 \%$ of the stars between $G=6$ and 16 can be used as primary stars, we find $w^{\text {Full }}=2420000 \mu \mathrm{as}^{-2}$, leading to $\sigma_{\gamma}^{\text {Full }}=1.3 \times 10^{-6}$. Including many more fainter stars gives only a marginal improvement. This estimated precision on PPN $\gamma$ is significantly better than today's best estimate from the Cassini mission $\left(2 \times 10^{-5}\right)$.

The small-scale simulations described above will in the future be calibrated against the results of large-scale solutions under development at the Gaia data processing center at ESAC (Madrid, Spain). Current solution runs with simulated observations for 2 million primary stars, based on the Besançon galaxy model (Robin et al. 2003), and the number of stars and the realism of the simulated data will successively increase in the next few years. Of greatest importance is that possible sources of systematic errors are carefully evaluated in subsequent studies.

Acknowledgements. The work of DH and LL is supported by the Swedish National Space Board. $\mathrm{BH}$ is an ELSA Fellow supported by the Marie Curie FP6 contract MRTN-CT-2006-033481.

\section{References}

de Bruijne, J. H. J. 2005, in: C. Turon, K. S. O'Flaherty, \& M. A. C. Perryman (eds.), The Three-Dimensional Universe with Gaia, ESA SP-576, p. 35

Hesteroffer, D., Mouret, S., Mignard, F., Tanga, P., \& Berthier, J. 2009, this proceedings, 325

Holl, B., Hobbs, D., \& Lindegren, L. 2009, this proceedings, 320

Frœschlé, M., Mignard, F., \& Arenou, F. 1997, in: B. Battrick, M. A. C. Perryman, \& P. L. Bernacca (eds.), HIPPARCOS Venice '97, ESA SP-402, p. 49

Lindegren, L., Høg, E., van Leeuwen, F., et al. 1992, A\&A A, 258, 18

Mignard, F. 2002, in: O. Bienaymé \& C. Turon (eds.), GAIA: A European Space Project, EAS Publications Series, Vol. 2 (EDP Sciences), p. 107

Mignard, F., Bailer-Jones, C., Bastian, U., et al. 2008, in: W. Jin, I. Platais, \& M. A. C. Perryman (eds.), A Giant Step: From Milli- to Micro-Arcsecond Astrometry, Proc. IAU Symposium No. 248 (Cambridge), p. 224

Robin, A. C., Reylé, C., Derriére, S., \& Picaud, S. 2003, A\&A, 409, 523

Will, C. M. 2006, The Confrontation between General Relativity and Experiment, Living Rev. Relativity 9, 3, URL (27-05-2009): http://www.livingreviews.org/lrr-2006-3/

Vecchiato, A., Lattanzi, M. G., Bucciarelli, B., Crosta, M., de Felice, F., \& Gai, M. 2003, A\& A 399,337 